\title{
Cécile RABOT, La Construction de la visibilité littéraire
}

\section{Monique Segré}

\section{OpenEdition}

\section{Journals}

Édition électronique

URL : https://journals.openedition.org/ress/3867

DOI : $10.4000 /$ ress. 3867

ISSN : 1663-4446

\section{Éditeur}

Librairie Droz

\section{Édition imprimée}

Date de publication : 25 mai 2018

Pagination : 283-286

ISSN : 0048-8046

\section{Référence électronique}

Monique Segré, «Cécile RABOT, La Construction de la visibilité littéraire », Revue européenne des sciences sociales [En ligne], 56-1 | 2018, mis en ligne le 22 juin 2017, consulté le 08 janvier 2022. URL : http:// journals.openedition.org/ress/3867 ; DOI : https://doi.org/10.4000/ress.3867

Ce document a été généré automatiquement le 8 janvier 2022

(C) Librairie Droz 


\title{
Cécile RABOT, La Construction de la visibilité littéraire
}

\author{
Monique Segré
}

\section{RÉFÉRENCE}

Cécile RABOT, 2015, La Construction de la visibilité littéraire, Villeurbanne, Presses de

l'Enssib, « Papiers », 392 p.

1 Avant d'aborder le travail de Cécile Rabot, donnons un bref aperçu de l'évolution des bibliothèques, de leurs objectifs et des publics auxquels elles s'adressent. Les bibliothèques furent d'abord des lieux assez austères destinés avant tout à la conservation des ouvrages réservés à des lecteurs lettrés et érudits. Ce n'est que vers les années 1960 qu'elles devinrent plus claires, plus attirantes et fréquentées par un public peu à peu plus diversifié. Cette lente ouverture fut aussi encouragée par les progrès de l'édition et la parution d'une multiplicité d'ouvrages de tous genres. De nombreuses bibliothèques municipales furent créées à partir des années 1960, passant de 500 à près de 1000 dans les années 1970 ; la lecture y fut encouragée, sollicitée par un personnel dûment formé (les écoles de bibliothécaires furent instaurées en 1963) désireux d'aider un public ayant peu à peu acquis le goût de la lecture. Les éditeurs soignèrent l'apparence du livre pour le rendre séduisant, donner l'envie de le saisir, le manipuler, le feuilleter, le parcourir afin que s'instaure le désir de s'en approprier le contenu lui-même agréablement typographié. Le «libre accès " fut instauré, censé permettre le libre choix ; cette liberté de choix se révéla trompeuse car elle mit et met encore aujourd'hui dans l'embarras les lecteurs désorientés : il faut savoir quoi choisir et comment, devant des rayons couverts d'une multitude de livres inconnus. Le « libre accès " n'est libre que pour ceux qui ont la connaissance du secret des critères de rangement. Rendre le livre accessible matériellement, bien que nécessaire, voire indispensable, ne permet pas de supprimer les obstacles intimes, mentaux (méconnaissance des auteurs proposés, sentiment d'incompétence ou d'indignité) qui rendent peu aisé le projet de lecture pour ceux qui ont une approche encore craintive 
et inusitée des bibliothèques. C'est pourquoi les bibliothèques restent fréquentées, en ce début $\mathrm{du} \mathrm{xxi}^{\mathrm{e}}$ siècle, essentiellement par les catégories sociales appartenant aux classes moyennes cultivées : $18 \%$ des français ont recours aux bibliothèques. «Par ses techniques d'offre comme par les ouvrages qu'elle offre la bibliothèque correspond d'abord aux attentes et aux pratiques des classes moyennes ", note Jean-Claude Passeron (Le Raisonnement sociologique. L'espace non-poppérien du raisonnement naturel, Paris, Nathan, 1991, p. 339).

2 Cécile Rabot a choisi de s'intéresser au plus près à l'offre en bibliothèques de romans de divertissement appréciés de ce public ; faciles à lire, favorisant l'évasion hors du réel et l'identification aux personnages, ces ouvrages apportent aussi des connaissances. Elle a pris le parti de chercher à comprendre comment sont choisis les romans ainsi proposés, quelles motivations animent les bibliothécaires responsables des sélections, comment ce personnel qualifié sait rendre « visibles » les ouvrages choisis, c'est-à-dire comment les mettre en valeur pour attirer le regard et susciter l'intérêt des futurs lecteurs. Il s'agit d'étudier en quoi l'offre est adaptée à leurs attentes et comment elle s'efforce de les séduire, de les encourager aussi à découvrir des auteurs qui jusque-là leur restaient inconnus, à susciter leur curiosité, à diversifier et accroître leurs intérêts, à les attirer vers des horizons qu'ils n'ont pas encore explorés.

3 Cette étude a incontestablement le mérite de la nouveauté. Elle permet de comprendre les conditions de la diversité de l'offre en bibliothèque ainsi que les modalités de cette offre en tenant compte simultanément de l'influence des médias, des intentions et des pratiques des bibliothécaires, des attentes et des comportements des usagers. Elle fourmille de remarques perspicaces tout en proposant au cours de son cheminement des résumés d'ouvrages pleins d'intérêt. Étude neuve mais souvent touffue et répétitive qui rend la démarche parfois difficile à cerner. Cécile Rabot a effectué une enquête auprès des bibliothécaires des soixante bibliothèques municipales de la ville de Paris, a participé au travail du comité de lecture, lu les compte rendus de réunions, analysé les entretiens avec des professionnels, pris connaissance des bibliographies, repéré les moyens variés de valorisation des ouvrages: présentoirs, listes bibliographiques, fascicules, animations. Quatre dispositifs ont plus particulièrement retenu son attention: les «coups de cœur», les «nouveautés», les "premiers romans» et les «mordus du polar».

4 C'est à juste titre que l'auteur a précisé le contexte éditorial des premières années 2000, période de l'étude; ces années sont en effet marquées par une production éditoriale croissante et foisonnante: 70000 nouveaux titres par an, chaque année 550 à 600 romans publiés à la rentrée de septembre et 500 en janvier. Les usagers des bibliothèques informés par les médias des publications récentes souhaitent emprunter les ouvrages aussitôt parus (par souci d'« être à la page ») ce qui est quasi impossible du fait des contraintes de fonctionnement (il faut le temps de la sélection des ouvrages, le temps de la commande, le temps du traitement: plastifier le livre, le pourvoir d'une cote, l'enregistrer dans une banque de données ; plusieurs mois peuvent s'écouler entre la décision d'achat et la mise en rayons).

5 Les ouvrages une fois sélectionnés et acquis par les bibliothèques, il convient de les rendre attrayants. Les bibliothécaires sont amenés à faire des choix tenant compte de leur pratique, de leur culture et de leur connaissance des critiques lues dans la presse spécialisée (Livres Hebdo surtout, magazines littéraires) ou dans la presse généraliste (Télérama, Le Nouvel Observateur), mais aussi de leur perception des attentes des habitués 
et de leur désir de faire découvrir aux usagers un nouvel univers car le bibliothécaire se veut à la fois un médiateur du livre mais aussi un passeur-découvreur capable de proposer au futur emprunteur des orientations de lecture qui lui sont inhabituelles. Le bibliothécaire s'informe donc, lit certaines des parutions selon le temps dont il dispose durant son temps privé (non son temps professionnel, du fait des nombreuses tâches qui l'accaparent), choisit les manières de solliciter l'attention, d'éveiller l'intérêt de ceux qui viennent chercher des livres en bibliothèque.

6 Les présentoirs sont un moyen essentiel pour capter le regard du futur emprunteur, d'où le souci de les placer dans l'entrée ou dans les espaces de circulation de la bibliothèque, de proposer la lecture de la première page de l'ouvrage, de la quatrième de couverture ou d'extraits du roman, de tenir compte de la taille, de la couleur ou de l'épaisseur du livre. Le nom de l'auteur est rendu bien visible et constitue un moyen d'accroche du regard. Un roman récemment paru voisine avec d'autres romans moins connus du même auteur afin de faire prendre conscience au public qu'il est peut-être en présence d'une œuvre. La mention des « prix grand public » accordés par des jurys non professionnels (grand prix des lectrices de Elle, «Goncourt des lycéens », prix des "mordus du polar" pour les adolescents) est aussi un stimulant de l'attention. Les bibliothécaires proposent également des " animations " pour encourager à la lecture, sous la forme de rencontres avec les auteurs, de conférences, de lectures orales, de mises en spectacles (les fables de La Fontaine, la légende des siècles de Victor Hugo, un documentaire sur Georges Perec); les adaptations au cinéma sont des moyens de provoquer le renouvellement de l'intérêt du futur lecteur sur des œuvres oubliées ou peu connues. Rabot met bien en évidence toutes les modalités subtiles et efficaces utilisées par les bibliothécaires afin d'attirer et réactiver la curiosité du lecteur potentiel.

7 L'exemplarité de l'étude de Cécile Rabot - c'est ce qui fait son apport essentiel - réside dans son souci de montrer les intentions affirmées des choix des bibliothécaires, leur bonne volonté culturelle (les bibliothécaires mentionnent souvent le bonheur qu'ils éprouvent à faire faire des découvertes aux emprunteurs) et les influences persistantes (pression médiatique, connaissance des habitudes parfois figées des usagers) qu'ils subissent dans leur mode de sélection des romans destinés à leurs lecteurs. Pourtant, les analyses de Rabot révèlent que les résultats des efforts des bibliothécaires ne sont pas à la hauteur de leurs espoirs; un monde sépare leurs intentions de leur pratique effective.

8 Rabot constate que dans les choix effectués prédominent les romans grand public, les best-sellers plébiscités par la presse et que les productions des éditeurs les plus marquants et reconnus restent les plus exposées. Ainsi les « coups de cœur » sont pour les trois quarts des publications de grands ou très grands éditeurs, ils ont déjà obtenu le succès des média et ont parfois fait l'objet d'une réédition en livres de poche. L'exposition sur présentoirs redouble une visibilité déjà construite par ailleurs. Il apparaît que les auteurs des «nouveautés " proposées sont déjà renommés et les choix des bibliothécaires viennent renforcer leur notoriété. La plupart du temps, malgré la bonne volonté des sélectionneurs qui affirment vouloir soutenir les auteurs obscurs, malgré leur désir de dénicher de nouveaux écrivains talentueux et de vouloir susciter des découvertes, les auteurs inconnus ou peu connus des bibliothécaires demeurent inaperçus, absents des présentoirs, voués à l'anonymat. C'est donc que les bibliothèques ont tendance à suivre ou à anticiper une demande largement conditionnée par les 
média plus qu'elles ne cherchent à la créer. Les pays dominants et les langues dominantes sont surreprésentés dans le monde de l'édition et en bibliothèques. Les États-Unis comptent pour $62 \%$ des littératures sélectionnées alors que l'Asie représente $9 \%$ de la sélection, l'Inde, $1 \%$, le Moyen Orient, $3 \%$, l'Amérique latine figurant dans les «nouveautés » au titre de $4 \%$ des littératures traduites. Les auteurs le plus souvent retenus dans les bibliothèques sont donc généralement français ou étasuniens: ils constituent $90 \%$ des nouveautés. Les "coups de cœur» comptent une minorité d'auteurs francophones (18\%) issus d'autres pays que la France. Même les premiers romans lesquels ne sont pourtant précédés d'aucune mention dans la presse, ne sont choisis que s'ils proviennent d'éditeurs français; les productions francophones ne figurent dans la sélection que si elles ont été publiées par un éditeur français.

9 La profusion des informations contenues dans cette étude est à souligner. Elle ne fait cependant pas oublier l'absence d'entretiens approfondis et analysés avec certains bibliothécaires et usagers, qui auraient contribué à éclaircir la compréhension des principaux résultats de cette recherche.

\section{AUTEURS}

\section{MONIQUE SEGRÉ}

CNRS, Laboratoire Georges-Friedmann - Université Paris-I 